\title{
Signal Reception for Space-Time Differentially Encoded Transmissions over FIR Rich Multipath Channels
}

\author{
Zhan Zhang \\ Department of Electrical and Computer Engineering, Dalhousie University, 1360 Barrington Street, P.O. Box 1000, \\ Halifax, NS, Canada B3J $2 X 4$ \\ Email: zhangz@dal.ca \\ Jacek llow \\ Department of Electrical and Computer Engineering, Dalhousie University, 1360 Barrington Street, P.O. Box 1000, \\ Halifax, NS, Canada B3J 2 X4 \\ Email: j.ilow@dal.ca
}

Received 1 January 2003; Revised 28 November 2003

\begin{abstract}
With sophisticated signal and information processing algorithms, air interfaces with space-time (ST) coding and multiple reception antennas substantially improve the reliability of wireless links. This paper proposes a new receiver algorithm for differential ST coded transmissions over the finite-impulse-response (FIR) rich multipath fading channels. The symbol detection introduced in this paper is a deterministic subspace-based approach in a multiple-input and multiple-output (MIMO) system framework. The receiver (i) operates in a blind fashion without estimating the channel or its inverse and (ii) is able to work with a small number of signal samples and hence can be applied in the quasistatic channels. The proposed scheme employs multiple antennas at both sides of the transceiver and exploits both the antenna diversity and the multiple constant modulus (MCM) characteristics of the signaling. The receiver is able to blindly mitigate the intersymbol interference (ISI) in a rich multipath propagation environment, and this has been verified through the extensive Monte Carlo simulations.
\end{abstract}

Keywords and phrases: rich multipath channels, space-time processing, transmit diversity, unitary group codes, signal subspace, constant modulus.

\section{INTRODUCTION}

Space-time (ST) multiple-input multiple-output (MIMO) transmission and reception is now regarded as one of the most effective approaches for increasing channel capacity or system fading-resistance $[1,2,3,4,5,6,7]$. Among a variety of ST coding schemes, differential ST modulation (DSTM) and differential space-code modulation (DSCM) are among the most promising ST coding schemes in wireless fading channels because of their efficient differential encoding and detection features $[8,9,10,11,12]$. Of particular interest to this paper is differential unitary group codes introduced in $[8,9,12]$.

The differential schemes can work whether the channel state information (CSI) is available or not, and this is what makes them very attractive. When an accurate estimation of the CSI is difficult or costly, the DSTM schemes are obviously preferable than other schemes which assume full knowledge of the CSI.

As a recent development, a new type of ST block code is the Khatri-Rao ST code (KRST) proposed in [13], which possesses a built-in channel identifiability. It relies on the blind identifiability properties of the trilinear models and parallel factor analysis to estimate the channel states and to detect the ST symbols. However, there are some concerns about the convergence speed of its iterative algorithm. DSTM does not have such an issue. Compared to DSTM, KRST has a higher computational complexity at the receiver. The DSTM was designed to maximize the diversity advantage of the code while maintaining a receiver implementation to be as simple as possible.

The common point of the DSTM, DSCM, and the KRST is that they all assume a frequency-flat fading channel modeling in their design and analysis. In this paper, we consider reception of the DSTM signals under more realistic and complex channel conditions in rich multipath environment. Multipath scattering and reflection effects characterize most wireless channels. They cause both time and angle spreads. As a result, most wireless channels are selective in time, space, and frequency, and this is a reason why this paper addresses multipath frequency-selective impairments in the design of the ST receiver. 
In contrast to the method presented in this paper, a combination of orthogonal frequency division multiplexing (OFDM) scheme with one of DSTM, DSCM, and KRST is feasible for transceiver designs over MIMO frequencyselective channels, because OFDM is capable of converting the frequency-selective channels into frequency-flat fading channels. Besides, to achieve the maximum diversity gain, a direct design of the frequency-ST coding scheme based on OFDM is also possible. An example is the transceiver proposed in [14]. However, the OFDM scheme has its own limitations: it has a very large peak to average power ratio, which demands a high linearity on the transmitter power amplifier. Nonlinearity of the system causes the intercarrier interference, which gives rise to the drastic degradation of the system performance. Moreover, performance of OFDM is more vulnerable to the frequency synchronization error than the conventional schemes, such as the single-carrier $M$-ary PSK, which the DSTM employs [15].

For channel equalizers requiring the channel estimation, the channel identification precision substantially affects the system performance. Small estimation bias may cause a severe performance degradation. In mobile communications, the channel changes quickly so that channel estimation is inefficient. Therefore, in this paper, channel estimation is neither assumed nor conducted in the algorithm. At the receiver, the transmitted data are recovered directly from the observed samples using an algebraic approach. Specifically, the new transceiver scheme consists of (i) a DSTM transmitter, (ii) an equalization algorithm based on direct input signal subspace estimation, and (iii) a differential ST symbol detector.

In general, the proposed receiver mitigates the multipath time-spread impairments without channel estimation provided that the channel is of rich multipath type so that its characterization matrix meets certain column-rank conditions. The approach used in this paper to recover the data relies on a modified version of signal subspace-based method introduced in [16]. The novelty of this paper stems from integrating subspace method based signal deconvolution and the exploitation of constant modulus property of the transmitted symbols to facilitate the noncoherent detection of DSTM signaling in a frequency-selective environment.

\section{REVIEW OF DIFFERENTIAL ST MODULATION}

In this section, the DSTM and unitary group codes [8] are briefly described for transmissions over frequency-flat fading channels. A transmitter equipped with $K$ antennas and a receiver equipped with $M$ antennas are assumed to constitute the transceiver system. A unitary ST codeword matrix $\mathbf{C}_{j}$ of size $K \times K$ is transmitted in the $j$ th time slot $T_{j}$ of duration $T_{c}=K \cdot T_{s}$, where $j$ is the time index and $T_{s}$ is the symbol duration. Each code matrix $\mathbf{C}_{j}$ is of the form $\mathbf{C}_{j}=\mathbf{C}_{j-1} \mathbf{G}_{j}$. Matrix $\mathbf{G}_{j}$ is chosen from a specific code set $g=\left\{\mathbf{G}_{(m)} \mid \mathbf{G}_{(m)} \mathbf{G}_{(m)}^{H}=\mathbf{I}\right\}$ to represent user data, where $m$ is the codeword index $(m=1,2, \ldots, \mathcal{M})$. The code has the property

$$
\mathbf{C}_{j} \mathbf{C}_{j}^{H}=K \mathbf{I}_{K \times K}
$$

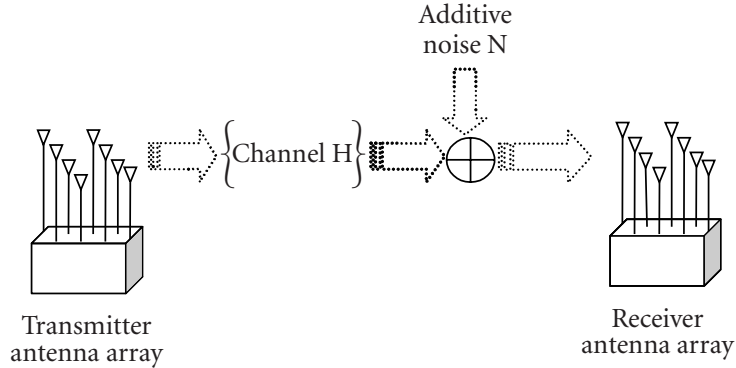

FIGURE 1: The general modeling of a multiantenna transceiver system.

It was proved in [9] that a full-rank unitary group code with $\mathcal{M}=2^{n}$ codewords is equivalent to either a cyclic group code or a dicyclic group code. Assuming that the unknown frequency-flat fading channel is characterized by matrix $\mathbf{H} \in$ $\mathcal{C}_{M \times K}$, the received data of the differentially ST coded signals at multiple receiving antennas are given as [8]

$$
\mathbf{Y}_{j}=\mathbf{H X}_{j}+\mathbf{N}_{j}
$$

where (i) the transmitted ST code is represented by $\mathbf{X}_{j}, j=$ $1,2, \ldots, J$; (ii) $J$ is a frame length in codewords; and (iii) $\mathbf{N}_{j}$ stands for the matrix version of additive white Gaussian noise (AWGN). With such modeling in a frequency-flat fading environment, a maximum likelihood (ML) decoder derived in $[8]$ is

$$
\widehat{\mathbf{G}}_{j}=\arg \max _{\mathbf{G}_{(m)}} \mathfrak{R}\left\{\operatorname{Tr}\left\{\mathbf{G}_{(m)} \mathbf{Y}_{j}^{H} \mathbf{Y}_{j-1}\right\}\right\},
$$

where $\mathfrak{R}$ stands for real part of the value and $\operatorname{Tr}$ denotes a trace computation. Hence, without knowing $\mathbf{H}$, the $\mathbf{G}_{j}$ can be estimated by observing the last two received data blocks $\left[\mathbf{Y}_{j-1}, \mathbf{Y}_{j}\right]$.

\section{THE NEW RECEIVER ALGORITHM FOR TRANSMISSION OVER FIR RICH MULTIPATH FADING CHANNELS}

\subsection{Basis representations of the transmitted signals}

In what follows, after a frame-by-frame DSTM transmitter is proposed, the discussion will focus on an equalization algorithm based on direct input signal subspace estimation.

The transmission scenario proposed in this paper for MIMO rich multipath channels is a frame-by-frame transmission/reception scheme illustrated in Figures 1 and 2, where $T_{c}$ is a time slot for a codeword and $T_{G} \geqslant L T_{s}$ is a frame guard interval to avoid the interframe interference ( $L$ is the maximum channel length of the subchannels).

At the receiver, the continuous-time received signal vector $Y(t)$ is sampled at the symbol rate $\left(1 / T_{s}\right)$ after down- 


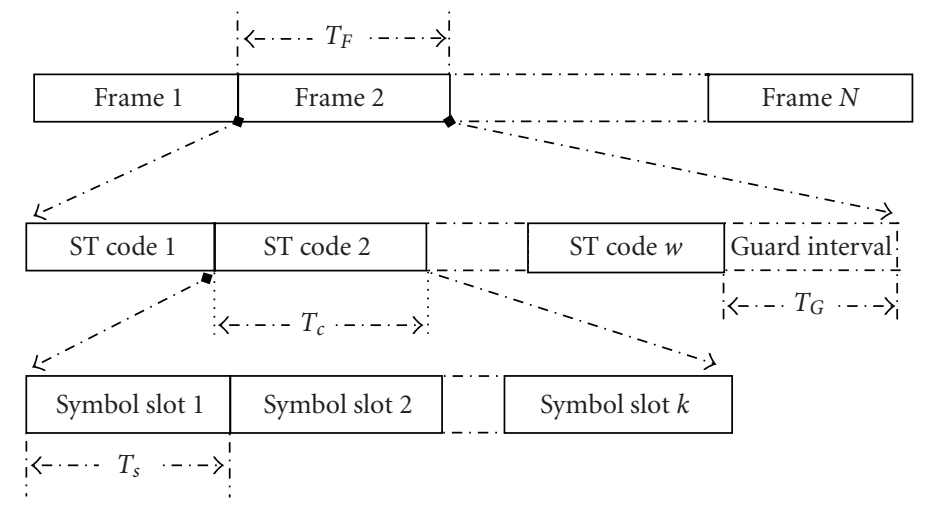

Figure 2: Transmitted signal frame structure and timing.

converting and reception filtering. For a period of signal frame $\left(T_{F}\right)$, the sampled data sequence of $Y(t)$ at a receiver is arranged in a matrix form as follows:

$$
\begin{aligned}
\mathbf{Y}_{M \times(N+L)} & =\left[\mathbf{y}_{0}, \mathbf{y}_{1}, \ldots, \mathbf{y}_{N+L-1}\right] \\
& =\left[\begin{array}{cccc}
y_{1}(0) & y_{1}(1) & \cdots & y_{1}(N+L-1) \\
y_{2}(0) & y_{2}(1) & \cdots & y_{2}(N+L-1) \\
\vdots & \vdots & \vdots & \vdots \\
y_{M}(0) & y_{M}(1) & \cdots & y_{M}(N+L-1)
\end{array}\right],
\end{aligned}
$$

where (i) $N$ is the frame length in symbols and (ii) $\mathbf{y}_{i}$ is a column vector of sampled data. We assume the quasistatic channel, namely, over the duration of one frame, the MIMO channel is time invariant.

According to the general modeling of MIMO channels, to capture the channel states, a matrix sequence $\{\mathbf{h}(i), i=$ $0,1, \ldots, L\}$ is used. If the noise effects are temporarily disregarded and with the proper arrangement of data, we get the following input-output relation in a matrix format for the qth frame:

$$
\mathbf{Y}_{M \times(N+L)}^{[q]}=\mathbf{H}_{M \times K(L+1)} \mathcal{X}_{K(L+1) \times(N+L)}^{[q]},
$$

where

$$
\begin{gathered}
\mathbf{H}_{M \times K(L+1)}=[\mathbf{h}(0), \mathbf{h}(1), \ldots, \mathbf{h}(L)] ; \\
X_{K(L+1) \times(N+L)}^{[q]} \\
=\left[\begin{array}{ccccccc}
\mathbf{x}(0) & \mathbf{x}(1) & \cdots & \mathbf{x}(N-1) & 0 & \cdots & 0 \\
0 & \mathbf{x}(0) & \cdots & \mathbf{x}(N-2) & \mathbf{x}(N-1) & \cdots & 0 \\
\vdots & \vdots & \vdots & \vdots & \vdots & \vdots & \vdots \\
0 & \cdots & 0 & \mathbf{x}(0) & \mathbf{x}(1) & \cdots & \mathbf{x}(N-1)
\end{array}\right]
\end{gathered}
$$

and $\mathbf{x}(i)$ is a column vector $\mathbf{x}(i)=\left[x_{1}(i), x_{2}(i), \ldots, x_{K}(i)\right]^{T}$.
In order to retrieve input (transmitted) signals from the observation of convoluted received signals, first, a matrix sequence $\left\{\mathfrak{Y}^{(p)} \mid p=0,1,2, \ldots, L\right\}$ is formed such that

$$
\mathfrak{Y}^{(p)}=\left[\mathbf{y}_{p}, \mathbf{y}_{p+1}, \ldots, \mathbf{y}_{p+N-1}\right] ; \quad p=0,1,2, \ldots, L,
$$

where $\mathfrak{Y}^{(p)}$ can be viewed as the vector subsequences of $\left[\mathbf{y}_{0}, \mathbf{y}_{1}, \ldots, \mathbf{y}_{N+L-1}\right]$ within a sliding window of width $N$ corresponding to the shift $p=0,1, \ldots, L$.

For every $\mathfrak{Y}^{(p)}$, we calculate a matrix $\Xi^{(p)}$ which consists of the spanning row vector set, that is, the rows of $\boldsymbol{\Xi}^{(p)}$ constitute the orthonormal basis for the subspace spanned by the rows of $\mathfrak{Y}^{(p)}$. The matrix $\Xi^{(p)}$ can be obtained by singular value decomposition (SVD) or some other efficient estimation methods. This processing is denoted in this paper by

$$
\mathfrak{Y}^{(p)} \Longrightarrow \boldsymbol{\Xi}^{(p)}, \quad p=0,1,2, \ldots, L .
$$

Proposition 1. Let the row vector subspace of $\mathbf{X}_{K \times N}=$ $\left[\begin{array}{llll}\mathbf{x}(0) & \mathbf{x}(1) & \cdots & \mathbf{x}(N-1)\end{array}\right]$ be denoted by $s_{X}$. In absence of the noise, the intersection of the row vector subspaces of $\boldsymbol{\Xi}^{(p)}$, $p=0,1, \ldots, L$, is equivalent to $s_{X}$ with a probability of 1 for transmissions employing unitary ST group codes, provided $\mathbf{H}$ is of a full-column rank and the signal frame length $N$ is sufficiently large for matrix $\mathbf{X}$ to have full-row rank.

The proof of Proposition 1 is in the appendix. The fullcolumn-rank assumption of $\mathbf{H}$ could be met with probability of 1 if it is a "tall" matrix with a row number larger than the column number if channel is of a rich multipath type. Evidently, if the subchannel lengths increase, accordingly, the number of reception antennas should be increased. Some auxiliary methods to facilitate meeting this assumption are discussed in Section 3.2. This assumption is a sufficient condition for the operation of the algorithm proposed in Section 4; however, it is not a necessary condition. 
As a matter of fact, for the proposed algorithm, it is only assumed that some matrices among $\mathbf{h}(i), i=0,1, \ldots, L$, individually have a full-column rank. This normally holds with probability of 1 for a rich multipath environment and the number of the reception antennas being larger than that of the transmission antennas. This assumption could be further relaxed by the data stacking method discussed in Section 3.2.

Defining a new matrix $\boldsymbol{\Xi}$ whose row vectors span the vector subspace intersection of $\boldsymbol{\Xi}^{(p)}, p=0,1, \ldots, L$, and denoting it by $\boldsymbol{\Xi}=\bigcap_{p=0}^{L} \boldsymbol{\Xi}^{(p)}$, from Proposition 1, we have that the rows of $\boldsymbol{\Xi}$ also span subspace $s_{X}$ with probability 1 . Therefore,

$$
\mathbf{X}_{K \times N}=\mathbf{W}_{K \times K} \boldsymbol{\Xi}_{K \times N}
$$

holds with probability 1 , where $\mathbf{W}_{K \times K}$ is a weight matrix. Hence, with a proper $\mathbf{W}$, the transmitted signals could be recovered completely from $\mathfrak{Y}^{(p)}$ by finding the spanning vector set using procedure of Proposition 1. In other words, the transmitted data could be recovered from $\mathfrak{Y}^{(p)}$ within the ambiguity of a transformation $\mathbf{W}$.

The above observation is a fundamental point for the receiver algorithm design in this paper based on direct input signal subspace estimation. The estimation of $\mathbf{W}$ will be discussed in Section 4.2.

\subsection{Column-rank assumption of channel matrices and oversampling}

Regarding the assumption for the column rank of $\mathbf{h}(i)$, the following discussion is in order. As analyzed in [17], rich multipath scattering normally causes wide angle spreads. In these situations, the channels can be modeled using uncorrelated high-rank matrices. For MIMO frequency-flat fading channels, a formula suggested in [17] to predict a high-rank channel situation is

$$
\frac{2 D_{t}}{K-1} \frac{2 D_{r}}{M-1}>\frac{R \lambda}{M}
$$

where (i) $D_{t}, D_{r}$ stand for the transmission and reception scattering radius, respectively; (ii) $R$ is the distance between transmitter and receiver; and (iii) $\lambda$ is the wavelength. This formula indicates that a large number of scatters (and large antenna spacing), large angle spreading, and small range $R$ will help in building up the high-rank MIMO channels in a frequency-flat fading modeling. High-rank MIMO channels can offer significant spatial multiplexing gain or diversity gain.

For MIMO frequency-selective channels, the above prediction method is applicable to channel matrices among $\mathbf{h}(i)$ that do not have zero columns. Therefore, it still brings insight to investigation of the MIMO frequency-selective channels and the scheme discussed in this paper.

To facilitate meeting the channel matrix column-rank requirements with minimum receiver antenna number, it is possible to arrange the received sample data for each frame by stacking the data $v$ times as follows:

$$
\begin{gathered}
\mathbb{Y}_{M v \times(N+L+v-1)}^{[q]}=\mathbb{H}_{M v \times K(L+v)} \mathbb{X}_{K(L+v) \times(N+L+v-1)}^{[q]}, \\
\mathbb{Y}^{[q]}=\left[\begin{array}{cccccccc}
\mathbf{y}_{0}^{[q]} & \mathbf{y}_{1}^{[q]} & \ldots & \cdots & \mathbf{y}_{N+L-1}^{[q]} & 0 & 0 \\
0 & \mathbf{y}_{0}^{[q]} & \mathbf{y}_{1}^{[q]} & \ldots & \mathbf{y}_{N+L-2}^{[q]} & \cdots & 0 \\
\vdots & \vdots & \vdots & \vdots & \vdots & \vdots & \vdots \\
0 & 0 & 0 & \mathbf{y}_{0}^{[q]} & \ldots & \cdots & \mathbf{y}_{N+L-1}^{[q]}
\end{array}\right], \\
\mathbb{H}=\left[\begin{array}{ccccccc}
\mathbf{h}(0) & \mathbf{h}(1) & \ldots & \ldots & \mathbf{h}(L) & 0 & 0 \\
0 & \mathbf{h}(0) & \mathbf{h}(1) & \cdots & \mathbf{h}(L-1) & \cdots & 0 \\
\vdots & \vdots & \vdots & \vdots & \vdots & \vdots & \vdots \\
0 & 0 & 0 & \mathbf{h}(0) & \cdots & \cdots & \mathbf{h}(L)
\end{array}\right],
\end{gathered}
$$

$\mathbb{X}^{[q]}=$

$$
\left[\begin{array}{ccccccc}
\mathbf{x}^{[q]}(0) & \mathbf{x}^{[q]}(1) & \cdots & \cdots & \mathbf{x}^{[q]}(N-1) & 0 & 0 \\
0 & \mathbf{x}^{[q]}(0) & \mathbf{x}^{[q]}(1) & \cdots & \mathbf{x}^{[q]}(N-2) & \cdots & 0 \\
\vdots & \vdots & \vdots & \vdots & \vdots & \vdots & \vdots \\
0 & 0 & 0 & \mathbf{x}^{[q]}(0) & \cdots & \cdots & \mathbf{x}^{[q]}(N-1)
\end{array}\right] .
$$

The arrangement of received data in the matrix above is different from that of [16] for improving signal detection at the first and last $L$ symbols in each transmitted frame.

If a large receive antenna number is not feasible, oversampling with larger reception bandwidth could be considered as an alternative approach to meet the necessary channel matrix rank condition. If the oversampling rate is $P,(P-1)$ times more data can be obtained and arranged as follows:

$$
\begin{aligned}
& {\left[\overline{\mathbf{y}}_{0}, \overline{\mathbf{y}}_{1}, \ldots, \overline{\mathbf{y}}_{N+L-1}\right]} \\
& =\left[\begin{array}{cccc}
\mathbf{y}(0) & \mathbf{y}(1) & \cdots & \mathbf{y}(N+L-1) \\
\mathbf{y}\left(\frac{1}{P}\right) & \mathbf{y}\left(1+\frac{1}{P}\right) & \cdots & \mathbf{y}\left(N+L-1+\frac{1}{P}\right) \\
\vdots & \vdots & \vdots & \vdots \\
\mathbf{y}\left(\frac{P-1}{P}\right) & \mathbf{y}\left(1+\frac{P-1}{P}\right) & \cdots & \mathbf{y}\left(N+L-1+\frac{P-1}{P}\right)
\end{array}\right],
\end{aligned}
$$

where the index $i+j / P$ stands for the $j$ th sample in the $i$ th symbol duration. Therefore, with a MIMO channel characterized by

$$
\begin{aligned}
& {[\overline{\mathbf{h}}(0), \overline{\mathbf{h}}(1), \ldots, \overline{\mathbf{h}}(L)]} \\
& =\left[\begin{array}{cccc}
\mathbf{h}(0) & \mathbf{h}(1) & \ldots & \mathbf{h}(L) \\
\mathbf{h}\left(\frac{1}{P}\right) & \mathbf{h}\left(1+\frac{1}{P}\right) & \ldots & \mathbf{h}\left(L+\frac{1}{P}\right) \\
\vdots & \vdots & \vdots & \vdots \\
\mathbf{h}\left(\frac{P-1}{P}\right) & \mathbf{h}\left(1+\frac{P-1}{P}\right) & \cdots & \mathbf{h}\left(L+\frac{P-1}{P}\right)
\end{array}\right],
\end{aligned}
$$


provided that the effects of transmission shaping filtering and reception filtering are encompassed into the channel $[\overline{\mathbf{h}}(0), \overline{\mathbf{h}}(1), \ldots, \overline{\mathbf{h}}(L)]$, the input-output relation in the oversampling case becomes

$$
\overline{\mathbb{Y}}_{P M v \times(N+L+v-1)}=\overline{\mathbb{H}}_{P M v \times K(L+v)} \mathbb{X}_{K(L+v) \times(N+L+v-1)},
$$

where

$$
\begin{aligned}
\overline{\mathbb{Y}} & =\left[\begin{array}{ccccccc}
\overline{\mathbf{y}}_{0}^{[q]} & \overline{\mathbf{y}}_{1}^{[q]} & \cdots & \cdots & \overline{\mathbf{y}}_{N+L-1}^{[q]} & 0 & 0 \\
0 & \overline{\mathbf{y}}_{0}^{[q]} & \overline{\mathbf{y}}_{1}^{[q]} & \cdots & \overline{\mathbf{y}}_{N+L-2}^{[q]} & \cdots & 0 \\
\vdots & \vdots & \vdots & \vdots & \vdots & \vdots & \vdots \\
0 & 0 & 0 & \overline{\mathbf{y}}_{0}^{[q]} & \cdots & \cdots & \overline{\mathbf{y}}_{N+L-1}^{[q]}
\end{array}\right], \\
\overline{\mathbb{H}} & =\left[\begin{array}{ccccccc}
\overline{\mathbf{h}}(0) & \overline{\mathbf{h}}(1) & \cdots & \cdots & \overline{\mathbf{h}}(L) & 0 & 0 \\
0 & \overline{\mathbf{h}}(0) & \overline{\mathbf{h}}(1) & \cdots & \overline{\mathbf{h}}(L-1) & \cdots & 0 \\
\vdots & \vdots & \vdots & \vdots & \vdots & \vdots & \vdots \\
0 & 0 & 0 & \overline{\mathbf{h}}(0) & \cdots & \cdots & \overline{\mathbf{h}}(L)
\end{array}\right],
\end{aligned}
$$

and $\mathbb{X}_{K(L+v) \times(N+L+v-1)}$ is the same as the one in (13).

In the oversampling case, it is possible to meet the fullrank requirement with a receiver antenna number smaller than that of transmitter antennas at the cost of oversampling complexity and wider reception bandwidth. The latter factor also causes degradation in signal-to-noise ratio (SNR) to a certain extent.

\section{ESTIMATION OF THE TRANSMITTED SIGNALS FROM RECEIVED DATA OVER RICH MULTIPATH CHANNELS IN THE PRESENCE OF NOISE}

The ST subchannels can be of different lengths and the signals are usually contaminated by the noise. In the presence of noise, $\delta_{X}$ may not necessarily be the subspace intersection of $\boldsymbol{\Xi}^{(p)}$ discussed in Section 3. However, it is still possible to search for independent vectors whose linear combinations can approximate row vectors in $\delta_{X}$ in a similar fashion. We propose the following algorithm for determining a spanning vector set from received signals to approximate the transmitted signal vectors. This scheme is verified to provide a robust performance through simulations, which is described in the next section.

\subsection{The basis estimation and approximation of transmitted signals}

In the description of the receiver algorithm, the following notation is adopted:

(a) $[\mathbf{A} ; \mathbf{B}]$ stands for a matrix formed by stacking matrices $\mathbf{A}$ and $\mathbf{B}$;

(b) $L$ is the maximum length of the MIMO subchannels and is assumed to be known to the receiver;

(c) $\left[n_{\eta}, \mathbf{q}\right]=\left.\cap_{i=1}^{i_{\max }}\left\{\boldsymbol{\Xi}^{(i)}\right\}\right|_{\eta}$ denotes the following computation routine:

(1) calculate SVD: $\mathbf{U} \boldsymbol{\Sigma} \mathbf{Q}=\operatorname{SVD}\left(\left[\boldsymbol{\Xi}^{(1)} ; \boldsymbol{\Xi}^{(2)} ; \ldots ; \boldsymbol{\Xi}^{\left(i_{\max }\right)}\right]\right)$, where $\mathbf{U}, \boldsymbol{\Sigma}$, and $\mathbf{Q}$ are the resulting matrices of the SVD computation;

(2) $\mathbf{q}=\mathbf{Q}_{\left[1: n_{\eta}:\right]}$, where $n_{\eta}$ is the number of singular vectors whose corresponding singular values are not less than $\eta \cdot \mathbf{Q}_{[a: b, ;]}$ denotes a matrix consisting of the rows from ath to $b$ th of matrix $\mathbf{Q}$. Parameters $\left[n_{\eta}, \mathbf{q}\right]$ are the computation results of this routine.

The proposed algorithm to estimate $s_{X}$ proceeds in three steps as follows.

Algorithm Procedure.

Step a

(1) $i_{\max }=L+v, r=0$;

(2) calculate $\left[n_{\eta}, \mathbf{q}\right]=\left.\cap_{i=1}^{i_{\max }}\left\{\boldsymbol{\Xi}^{(i)}\right\}\right|_{\eta=0.96\left(\lambda_{\max }-1\right)}$, where $\lambda_{\max }$ is the current largest singular value;

(3) $\mathbf{V}^{(r)}=\mathbf{q} ; r+1 \Rightarrow r$;

(4) if $n_{\eta}<K$, go to Step b; else go to Step c.

Step $b$ if $i_{\max }>1$,

(1) $i_{\max }=i_{\max }-1$

(2) calculate $\left[n_{\eta}, \mathbf{q}\right]=\left.\bigcap_{i=1}^{i_{\max }}\left\{\boldsymbol{\Xi}^{(i)}\right\}\right|_{\eta=0.96\left(\lambda_{\max }-1\right)}$, where $\lambda_{\max }$ is the current largest singular value;

(3) $\mathbf{V}^{(r)}=\mathbf{q} ; r+1 \Rightarrow r$;

(4) if $n_{\eta}<K$, repeat Step b, else go to Step c; else go to Step c.

Step c

(1) Calculate $\left[n_{\eta}, \mathbf{q}\right]=\left.\bigcap_{i=1}^{r}\left\{\mathbf{V}^{(i)}\right\}\right|_{\eta=0.96}$;

(2) $\hat{\Xi}=\mathbf{q}$.

In the above computation of the intersection of basis vectors by SVD analysis, $\lambda_{\max }$ is an important parameter because it is used to compute how many $\boldsymbol{\Xi}^{(i)}$ share certain vectors as row basis vector. Computing and applying $\lambda_{\max }$ at each step instead of setting a constant value makes the algorithm adaptive to different channel-rank situations.

In the presence of noise and channel-rank deficiency, the above basis-vector searching algorithm may get more vectors than the desired basis vectors as computation results. However, this does not prevent approximating the transmitted signals. In this paper, the signaling property of multiple constant modulus (MCM) is taken advantage of to properly weight the estimated basis in approximating the original signal vectors. The "closer" vectors to the original signal vector basis are sorted out by their dominant weights obtained from the MCM constraint.

Specifically, once the matrix $\hat{\boldsymbol{\Xi}}$ is obtained, the transmitted signal matrix $\mathbf{X}_{K \times N}$ can be approximated by exploiting the MCM property. Similarly as in (9), the relation between $\hat{\mathbf{X}}_{K \times N}$ and $\hat{\Xi}_{S \times N}$ can be expressed as follows:

$$
\widehat{\mathbf{X}}_{K \times N}=\widehat{\mathbf{W}}_{K \times S} \hat{\Xi}_{S \times N},
$$

where (i) $\hat{\Xi}$ stands for a matrix whose row vectors are the estimated bases and (ii) $\hat{\mathbf{X}}$ represents the estimate of signal frame after deconvolution. The number of row vectors in $\hat{\boldsymbol{\Xi}}$ may be greater than the number of the signal vector basis due to the noise effects. Hence, the matrix $\widehat{\mathbf{W}}$ is not necessarily a square matrix as $\mathbf{W}$ in (9).

The noise components have direct influences on the algorithm in two aspects: (i) the noise degrades the estimation 
accuracy of the $\boldsymbol{\Xi}$ and $\mathbf{W}$; (ii) the random noise makes the processing time of each estimation vary from frame to frame. The sensitivity of the algorithm to the noise was examined by the simulations elaborated in Section 5.

The weight matrix $\widehat{\mathbf{W}}$ is calculated using the alternating projection iterations algorithm presented in the next section.

\subsection{Signal property projection}

DSTM employs PSK signaling so that transmitted signals have MCM characteristics. Therefore, an alternating projection method from [18] is adopted here to calculate $\widehat{W}$ in the following procedure.

Algorithm Procedure. For $j=0,1, \ldots, n$,

(1) $\hat{\mathbf{X}}_{K \times N}^{(j)}=\widehat{\mathbf{W}}_{K \times S}^{(j)} \hat{\boldsymbol{\Xi}}_{S \times N}$,

(2) $\tilde{\mathbf{X}}_{K \times N}^{(j)}=$ Proc_G_S $\left\{\hat{\mathbf{X}}^{(j)}\right\}$,

(3) $\overline{\mathbf{X}}^{(j)}=\boldsymbol{\lambda}^{(j)} \hat{\mathbf{X}}^{(j)}+\left(\mathbf{I}-\boldsymbol{\lambda}^{(j)}\right) \tilde{\mathbf{X}}^{(j)}$,

(4) $\overline{\mathbf{X}}^{(j+1)}=\overline{\mathbf{X}}^{(j)} \cdot /\left|\overline{\mathbf{X}}^{(j)}\right|$,

(5) $\widehat{\mathbf{W}}_{K \times S}^{(j+1)}=\overline{\mathbf{X}}_{K \times N}^{(j+1)} \hat{\Xi}_{S \times N}^{\dagger}$,

where Proc_G_S means the Gram-Schmidt orthogonalization procedure, and $\boldsymbol{\lambda}^{(j)}$ is a diagonal relaxation matrix. The initial matrix $\widehat{\mathbf{W}}^{(0)}$ could be either determined by pilot signals or choosing randomly a full-column-rank matrix. As mentioned in [18], the Gram-Schmidt orthogonalization procedure is applied here to prevent the algorithm from being biased to certain signals of strong power. The iteration stops when $\widehat{\mathbf{W}}^{(j)}$ reaches a stable state, that is, norm $\left(\widehat{\mathbf{W}}^{(j+1)}\right.$ $\left.\widehat{\mathbf{W}}^{(j)}\right) \leq \varepsilon$, where $\varepsilon$ is a small constant.

\subsection{Signal detection}

After the $\widehat{W}$ is estimated by the above procedure, the transmitted signal could be approximated as in (18). The relation between the original coded signal frame $\mathbf{X}=$ $\left[\mathbf{x}_{1}, \mathbf{x}_{2}, \mathbf{x}_{3}, \ldots, \mathbf{x}_{c}\right]$ and the estimate $\hat{\mathbf{X}}=\left[\widehat{\mathbf{x}}_{1}, \widehat{\mathbf{x}}_{2}, \widehat{\mathbf{x}}_{3}, \ldots, \widehat{\mathbf{x}}_{c}\right]$ can be modeled as

$$
\begin{gathered}
\hat{\mathbf{X}}=\mathbf{A X}+\mathbf{N} \\
\widehat{\mathbf{x}}_{i}=\mathbf{A x}_{i}+\mathbf{n}_{i}, \quad i=1,2, \ldots, c,
\end{gathered}
$$

where $\mathbf{A}$ is an admissible matrix and $\mathbf{x}_{i}$ is an ST group code matrix. Noise elements are assumed to have independent and identical circularly symmetric complex Gaussian distribution $\mathcal{C} \mathcal{N}\left(0, \delta^{2}\right)$.

Definition 1 (see [18]). If $\alpha_{k} \in\left\{\alpha|| \alpha_{k} \mid=1, k=1, \ldots, d\right\}$ $\subset \mathcal{C}$ and $\mathbf{P}$ is a permutation matrix, the matrix $\mathbf{A}=$ $\left(\operatorname{diag}\left(\alpha_{1}, \alpha_{2}, \ldots, \alpha_{d}\right) \mathbf{P}\right)$ is an admissible transformation matrix.

The ambiguity between $\mathbf{X}$ and its estimate $\hat{\mathbf{X}}$, represented by $\mathbf{A}$, exists because the MCM signal property constraint used in estimating $\widehat{\mathbf{W}}$ does not contain any phase information. From equations

$$
\begin{aligned}
\hat{\mathbf{x}}_{i}=\mathbf{A} \mathbf{x}_{i}+\mathbf{n}_{i}, & \hat{\mathbf{x}}_{i+1}=\mathbf{A} \mathbf{x}_{i+1}+\mathbf{n}_{i+1}, \\
\mathbf{x}_{i+1}= & \mathbf{x}_{i} \mathbf{G}_{[m]},
\end{aligned}
$$

we obtain the following relations:

$$
\widehat{\mathbf{x}}_{i+1}=\widehat{\mathbf{x}}_{i} \mathbf{G}_{[m]}+\ddot{\mathbf{n}}_{i+1},
$$

where

$$
\ddot{\mathbf{n}}_{i+1}=\mathbf{n}_{i+1}-\mathbf{n}_{i} \mathbf{G}_{[m]} .
$$

The dependence between $\widehat{\mathbf{x}}_{i+1}$ and $\widehat{\mathbf{x}}_{i}$ indicates a differential relation with the multiplicative matrix $\mathbf{G}_{[m]}$. It can be observed that the ambiguity matrix A between $\widehat{\mathbf{x}}_{i}$ and $\mathbf{x}_{i}$ is removed by the differential signaling and differential detection.

Hence, the detection of $\mathbf{G}^{[i]}$ can be carried out using a least square error detector:

$$
\widehat{\mathbf{G}}^{[i+1]}=\arg \min _{\mathbf{G}_{[r]}}\left\|\widehat{\mathbf{x}}_{i+1}-\widehat{\mathbf{x}}_{i} \mathbf{G}_{[r]}\right\|,
$$

where, for the $\mathbf{G}$ matrices, the matrix subscript $r$ is an ST codeword alphabet index, and the superscript $i$ is a time index of the ST codeword.

From (24), we get

$$
\begin{aligned}
\hat{\mathbf{G}}^{[i+1]}=\arg \min _{\mathbf{G}_{[r]}} \operatorname{Tr}\left\{\left(\widehat{\mathbf{x}}_{i+1}-\widehat{\mathbf{x}}_{i} \mathbf{G}_{[r]}\right)^{H}\left(\widehat{\mathbf{x}}_{i+1}-\widehat{\mathbf{x}}_{i} \mathbf{G}_{[r]}\right)\right\} \\
=\arg \min _{\mathbf{G}_{[r]}} \mathbf{T r}\left\{\widehat{\mathbf{x}}_{i+1}^{H}\left(\widehat{\mathbf{x}}_{i+1}\right)-\left(\widehat{\mathbf{x}}_{i} \mathbf{G}_{[r]}\right)^{H} \widehat{\mathbf{x}}_{i+1}\right. \\
\left.-\left(\widehat{\mathbf{x}}_{i+1}\right)^{H}\left(\widehat{\mathbf{x}}_{i} \mathbf{G}_{[r]}\right)+\left(\widehat{\mathbf{x}}_{i} \mathbf{G}_{[r]}\right)^{H}\left(\widehat{\mathbf{x}}_{i} \mathbf{G}_{[r]}\right)\right\} .
\end{aligned}
$$

Because $\operatorname{Tr}\left\{\left(\hat{\mathbf{x}}_{i} \mathbf{G}_{[r]}\right)^{H}\left(\widehat{\mathbf{x}}_{i} \mathbf{G}_{[r]}\right)\right\}$ is a constant for different $\mathbf{G}_{[r]}$, the detector for DSTM's differential signaling becomes

$$
\widehat{\mathbf{G}}^{[i+1]}=\arg \max _{\mathbf{G}_{[r]}} \mathfrak{R}\left\{\mathbf{T r}\left\{\left(\widehat{\mathbf{x}}_{i} \mathbf{G}_{[r]}\right)^{H}\left(\widehat{\mathbf{x}}_{i+1}\right)\right\}\right\} .
$$

Through the approximation of the signals with the estimated basis as in (18), the intersymbol interference (ISI) of the signal is mitigated. Hence, in the procedure proposed in this paper for MIMO frequency-selective channels, the final detection stage embodied through (26) is similar to that for DSTM signaling over frequency-flat fading MIMO channels as represented in (3). In the comparison of (26) and (3), the following property is useful: for square matrices $\mathbf{A}$ and $\mathbf{B}$, $\operatorname{Tr}\{\mathbf{A B}\}=\operatorname{Tr}\{\mathbf{B A}\}$.

\subsection{Summary of the receiver algorithm}

The complete receiver algorithm proposed for DSTM signaling over the finite-impulse-response (FIR) rich multipath channels proceeds on a frame-by-frame basis according to the following four steps:

(1) estimate the direct input signal subspace basis and signal approximations according to the method in Section 3.1;

(2) calculate $\widehat{W}$ by iterating the alternating projections exploiting MCM using the algorithm presented in Section 3.2;

(3) determine $\hat{\mathbf{X}}$ by $\widehat{\mathbf{X}}=\widehat{\mathbf{W}} \hat{\Xi}$; 


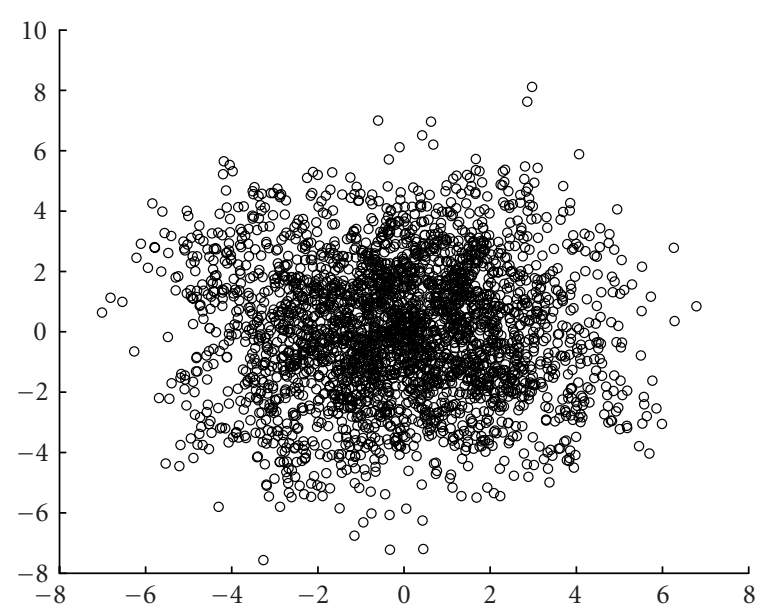

Figure 3: Received signal constellation diagram $(L=7, M=6$, $K=4, P=1, N=256, \mathrm{SNR} /$ bit/antenna $=18.5 \mathrm{~dB})$.

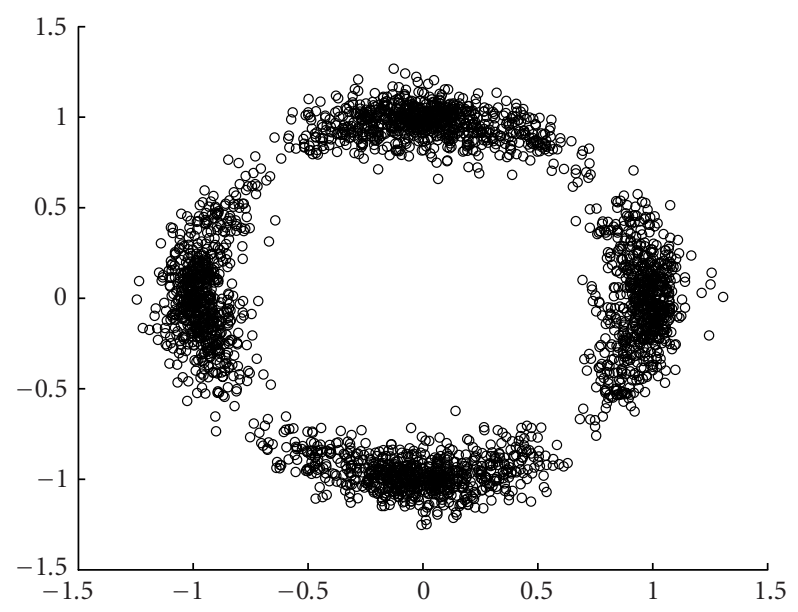

FIgURE 4: Signal constellation diagram after equalization $(L=7$, $M=6, K=4, P=1, N=256, \mathrm{SNR} /$ bit/antenna $=18.5 \mathrm{~dB})$.

(4) perform signal detection according to detection criteria (26) as described in Section 4.3.

Provided that the maximum delay spread is less than $T_{G}$, the block Toeplitz signal structure and data processing procedures in Sections 3.1, 3.2, and 4.3 enable the algebraic data recovery without channel knowledge and channel estimation. The procedures in Sections 3.1 and 3.2 mitigate frequency-selective effects in rich multipath environment, and the differential detection of ST symbols described in Section 4.3 removes the ambiguity of transformation $\mathbf{A}$ in (19).

Regarding the proposed algorithm, it should be noted that the receiver algorithm proposed in this paper exploits both block Toeplitz structure of the received signals and the MCM property of $M$-ary PSK signaling. It is not directly applicable to the schemes with a signaling without constant envelope. When employing other signalings that do not have

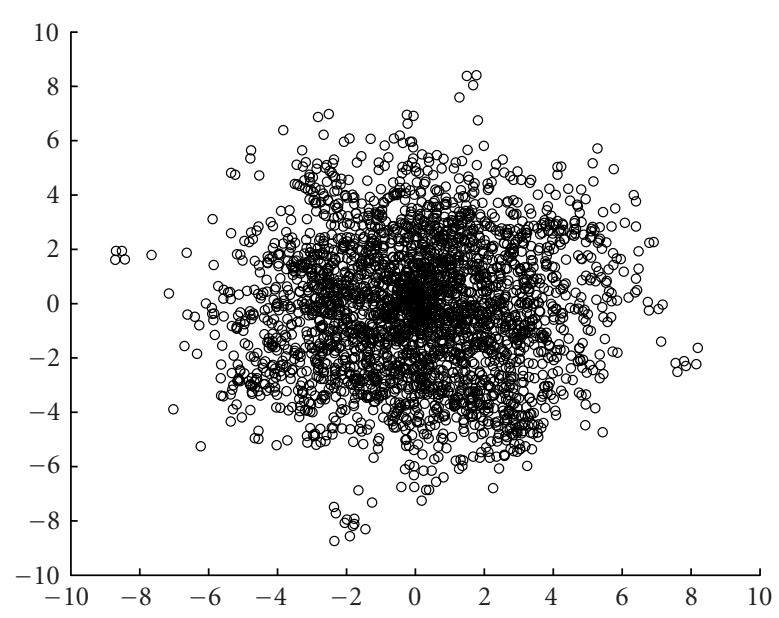

FIgURE 5: Received signal constellation diagram $(L=7, M=6$, $K=4, P=1, N=256$, SNR/bit/antenna $=19.3 \mathrm{~dB})$.

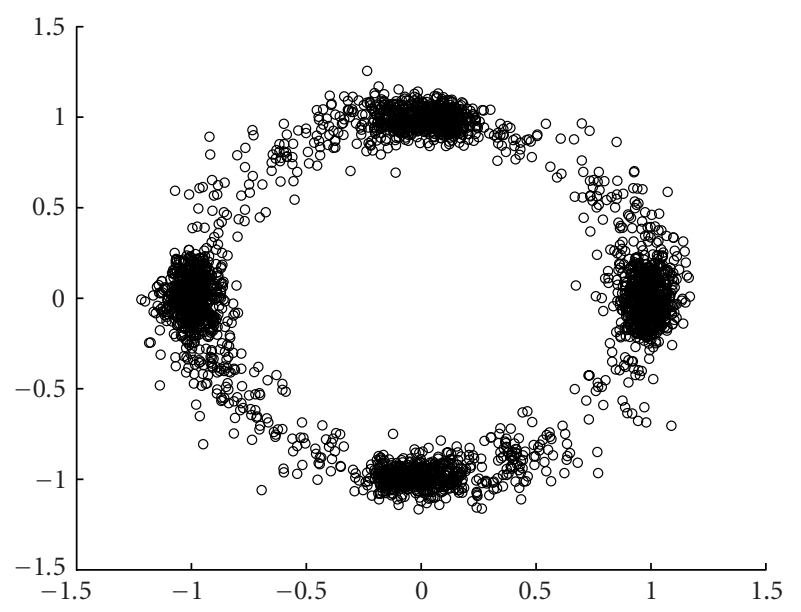

FIGURE 6: Signal constellation diagram after equalization $(L=7$, $M=6, K=4, P=1, N=256, \mathrm{SNR} /$ bit/antenna $=19.3 \mathrm{~dB})$.

the MCM property, the part of the receiver algorithm described in Section 3.2 for estimating $\mathbf{W}$ must be modified.

\section{PERFORMANCE SIMULATIONS}

With different parameter settings of the transceiver and the channels, simulations of the new receiver algorithm were conducted to verify the bit error rate (BER) performance over Rayleigh FIR fading channels in the presence of AWGN. Figures $3,4,5,6,7$, and 8 illustrate the signal constellation before and after the equalization for different values of SNR per antenna. From Figures 4, 6, and 8, it is evident that enforcing the MCM property in our algorithm causes the signal constellation after equalization to have a circular appearance.

The representative BER simulation results with the parameters $K=4, M=5,6, N=256$, and $P=1$ are illustrated in Figures 9, 10, and 11 for $L=3,5,7$, respectively. 


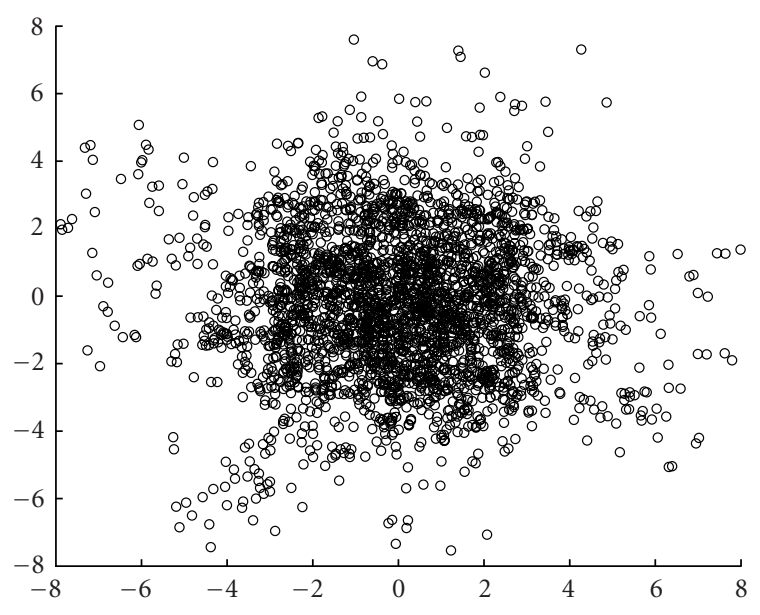

Figure 7: Received signal constellation diagram $(L=7, M=6$, $K=4, P=1, N=256, \mathrm{SNR} /$ bit/antenna $=21.4 \mathrm{~dB})$.

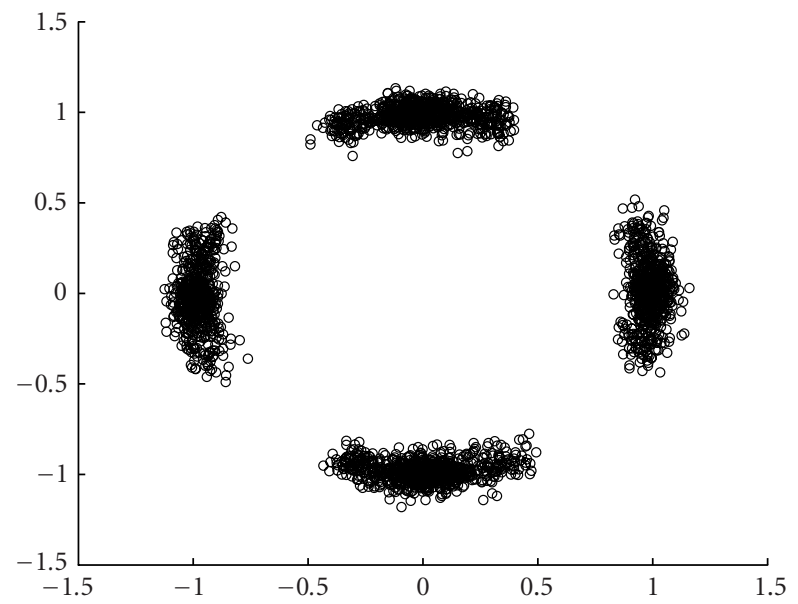

FIgURE 8: Signal constellation diagram after equalization $(L=7$, $M=6, K=4, P=1, N=256, \mathrm{SNR} /$ bit/antenna $=21.4 \mathrm{~dB})$.

The multiple channels were simulated to be the FIR Rayleigh fading channels.

The simulations were carried out by employing a $\left(\mathcal{M} ; k_{1}, \ldots, k_{4}\right)=(4 ; 1,1,1,1)$ cyclic group code [9] and QPSK signaling. The results were statistically averaged over all possible cases of random path delays of the subchannels, random ST channel states, random bitstreams, and random AWGN. The SNR values in Figures 9, 10, and 11 are the spatially and temporally averaged SNR per antenna over all the frames received.

For comparison purposes, the performance of DSTM signaling with the previous receiver's algorithm was simulated with the same fading channels. From the figures, it can be observed that the receiver (without equalization) derived under the assumption of the frequency-flat fading channels fail in the frequency-selective fading channels considered in the simulations (curves are labeled as "without equalization" in the figures). On the other hand, the proposed algorithm

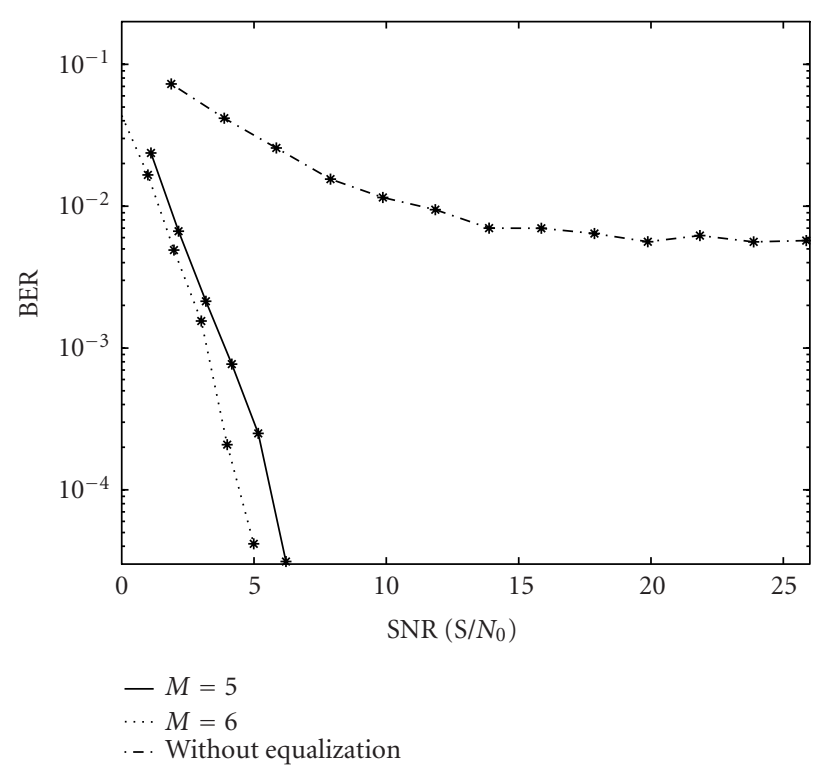

FIGURE 9: System BER performance in time-dispersive fading channel $(L=3, K=4, P=1, N=256)$.

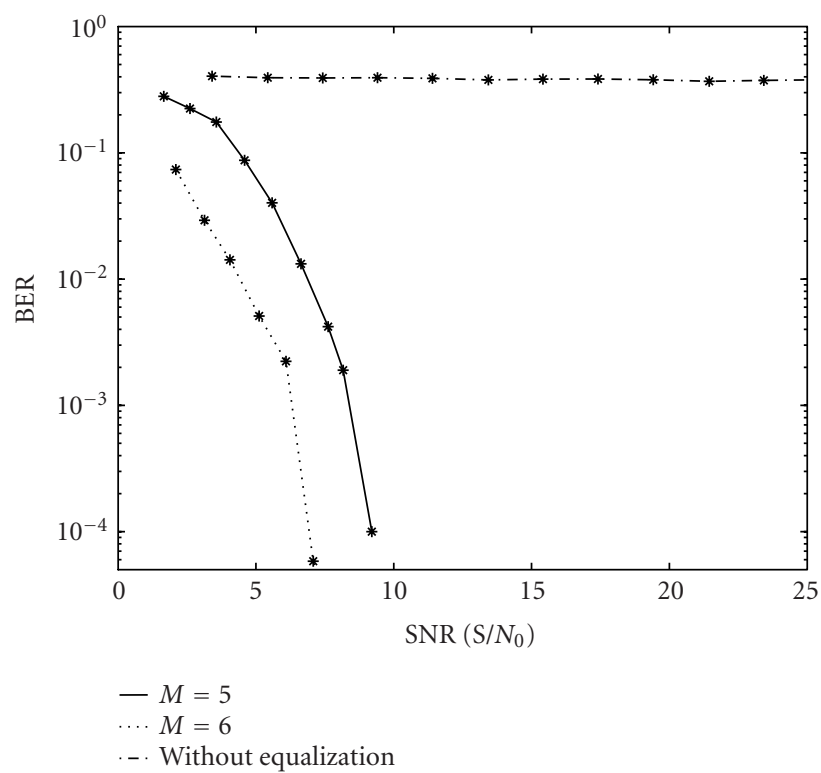

FIGURE 10: System BER performance in time-dispersive fading channel $(L=5, K=4, P=1, N=256)$.

(with equalization) maintains a robust performance in rich multipath quasistatic FIR fading channels.

When the channel length is increased, it is more difficult to remove the ISI effects. This is evident by comparing the performance curves in Figures 9, 10, and 11, where $L=3,5$, and 7, respectively. From these figures, we can observe that in order to obtain the same performance of BER at $10^{-3}$ using the same transceiver setup, the SNR has to be increased from $4 \mathrm{~dB}$ to $7 \mathrm{~dB}$ and $14.1 \mathrm{~dB}$ for $K=4, M=5$. Additionally, 


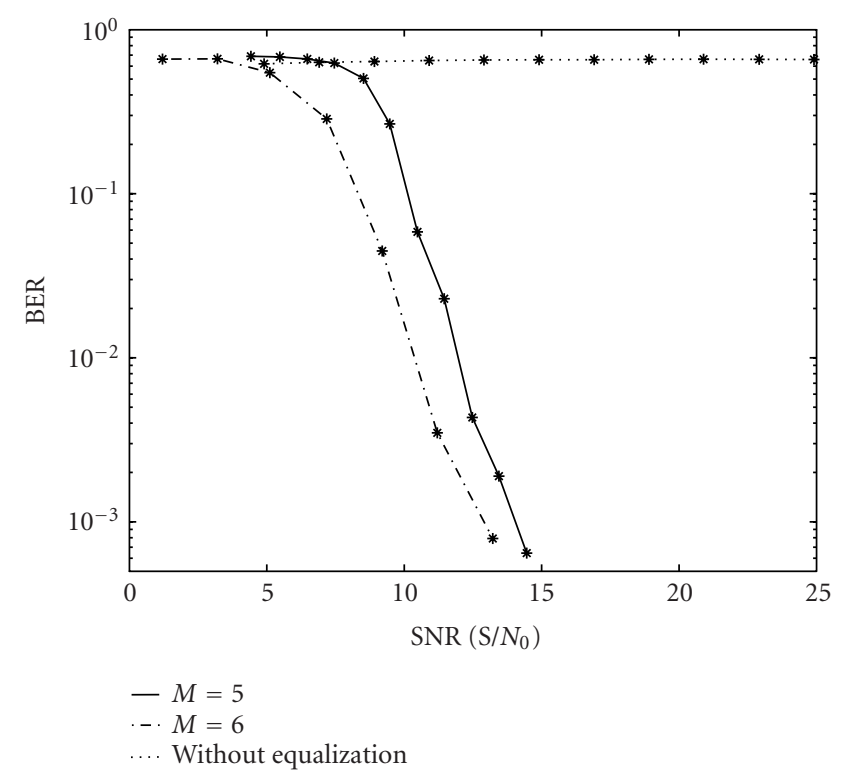

FIGURE 11: System BER performance in time-dispersive fading channel $(L=7, K=4, P=1, N=256)$.

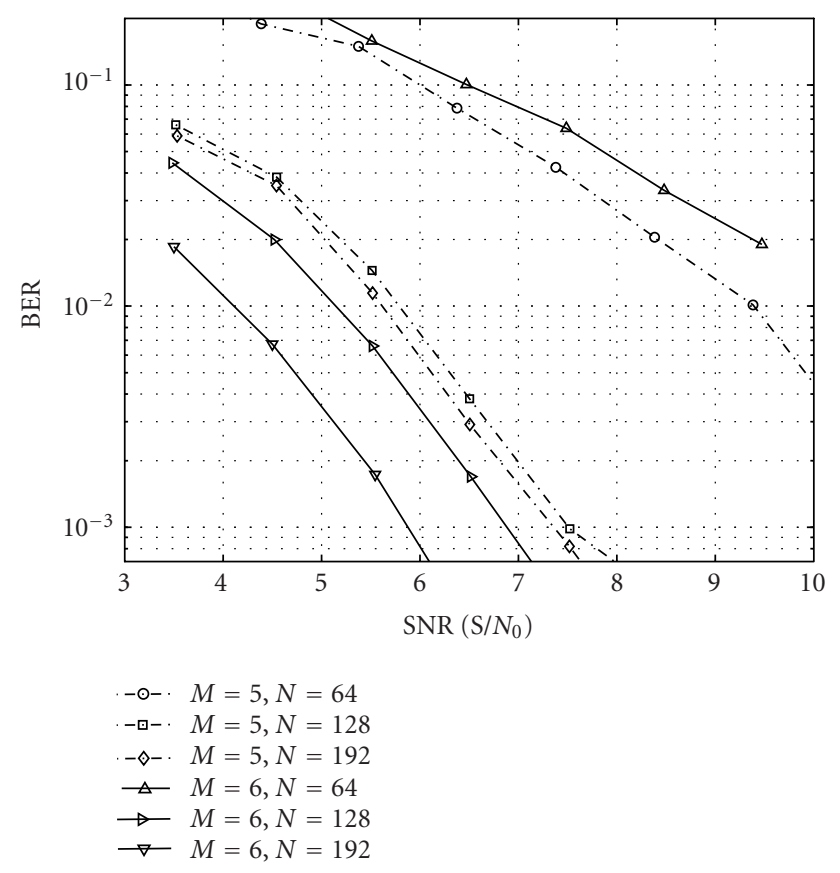

FIgURE 12: System BER performance in time-dispersive fading channel $(L=5, K=4, M=5,6, P=1)$.

the power savings by increasing the receiver antenna number depends on the BER operating point of the system.

Similarly, for different $N=64,128,192$, the simulation results with the parameters $K=4, M=5,6$, and $P=1$ are illustrated in Figures 12, 13, and 14 for $L=5,6,7$, respectively. From these figures, we could observe that the choices of $\mathrm{N} \mathrm{ex}-$ hibit a considerable influence on the system performance. To

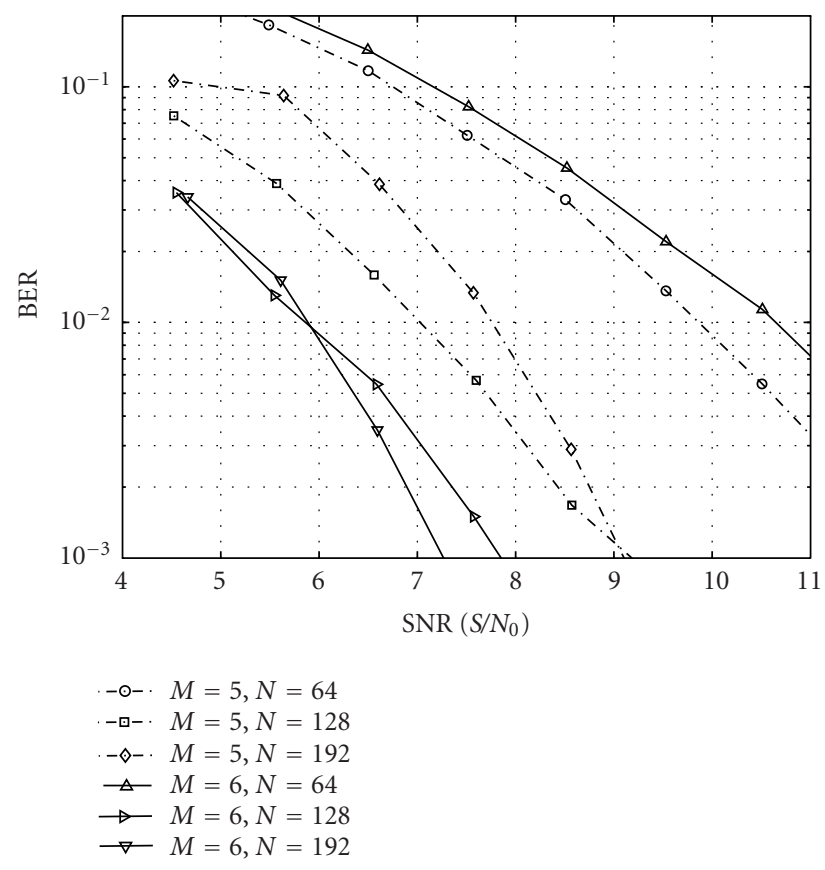

FIGURE 13: System BER performance in time-dispersive fading channel $(L=6, K=4, M=5,6, P=1)$.

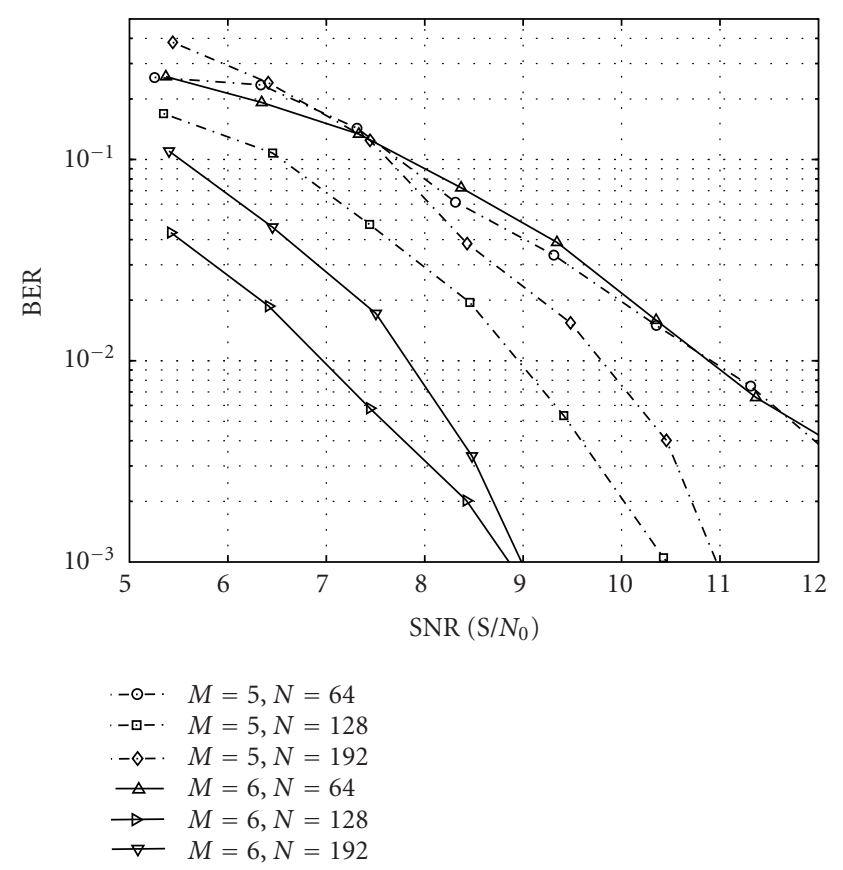

FIGURE 14: System BER performance in time-dispersive fading channel $(L=7, K=4, M=5,6, P=1)$.

some extent, for short channel length cases, a relatively larger $N$ within a certain range facilitates higher performance. The improvements are achieved at the expense of the increased computational complexity. But, for the cases of long channel lengths, this trend does not exist. 


\section{CONCLUSIONS}

This paper proposes a blind ST receiver algorithm for DSTM transmissions over quasistatic FIR fading channels. The algorithm is applicable in the transmission scenarios with different numbers of antennas at both the transmitter and receiver sides. Simulation results demonstrate its robust performance over unknown rich multipath FIR fading channels. With a proper design of the transceiver parameters and the frame guard time $T_{G}$ in the new scheme, the ST symbol detection error drops significantly when SNR passes certain thresholds despite the delay spread of the channels.

Particularly, the new detection algorithm does not rely on the channel estimation. Secondly, the proposed receiver is not subjected to the channel changes provided the channel is invariant within one frame time slot. Furthermore, in contrast to the methods based on the statistics of a large amount of signal samples, the proposed scheme is capable of operating when a relatively small number of received data samples are available.

\section{APPENDIX}

\section{PROOF OF PROPOSITION 1}

Proof. Let $\delta_{\mathfrak{Y}(p)}$ denote the row span of $\boldsymbol{\Xi}^{(p)}, p=0,1,2, \ldots, L$. If $\mathbf{H}$ is of a full-column rank, from (5), it could be concluded that

For $p=0$,

$\oint_{\mathfrak{Y}^{(0)}}=$

row_span $\left\{\begin{array}{ccccccc}\mathbf{x}(0) & \mathbf{x}(1) & \mathbf{x}(2) & \mathbf{x}(3) & \cdots & \cdots & \mathbf{x}(N-1) \\ 0 & \mathbf{x}(0) & \mathbf{x}(1) & \mathbf{x}(2) & \cdots & \cdots & \mathbf{x}(N-2) \\ \vdots & \vdots & \vdots & \vdots & \vdots & \vdots & \vdots \\ 0 & \cdots & 0 & \mathbf{x}(0) & \mathbf{x}(1) & \cdots & \mathbf{x}(N-L-1)\end{array}\right\} ;$

For $p=1$,

$\wp_{\mathfrak{Y}^{(1)}}$

=row_span $\left\{\begin{array}{cccccc}\mathbf{x}(1) & \mathbf{x}(2) & \cdots & \cdots & \mathbf{x}(N-1) & 0 \\ \mathbf{x}(0) & \mathbf{x}(1) & \cdots & \cdots & \cdots & \mathbf{x}(N-1) \\ \vdots & \vdots & \vdots & \vdots & \vdots & \vdots \\ 0 & \cdots & \mathbf{x}(0) & \mathbf{x}(1) & \cdots & \mathbf{x}(N-L)\end{array}\right\}$;

$$
\text { For } p=L
$$

$\oint_{\mathfrak{Y}^{(L)}}=$ row_span

$\times\left\{\begin{array}{cccccc}\mathbf{x}(L) & \mathbf{x}(L+1) & \cdots & \mathbf{x}(N-1) & 0 & 0 \\ \mathbf{x}(L-1) & \mathbf{x}(L-2) & \cdots & \cdots & \mathbf{x}(N-1) & 0 \\ \vdots & \vdots & \vdots & \vdots & \vdots & \vdots \\ \mathbf{x}(0) & \mathbf{x}(1) & \mathbf{x}(2) & \cdots & \cdots & \mathbf{x}(N-1)\end{array}\right\}$.
By observing the above relationship, it is evident that $\wp_{X} \subset$ $\varsigma_{\mathfrak{Y}^{(i)}}$, respectively, for $i=0,1,2, \ldots, L$. Therefore, according to set theory,

$$
s_{X} \subset\left\{\bigcap_{i=0}^{L} s_{\mathfrak{Y}^{(i)}}\right\} .
$$

Consider $\varsigma_{\mathfrak{Y}^{(1)}} \cap \varsigma_{\mathfrak{Y}^{(2)}}$, which is equivalent to the intersection of row subspaces of

$$
\begin{gathered}
\left\{\begin{array}{cccccccc}
\mathbf{x}(0) & \mathbf{x}(1) & \mathbf{x}(2) & \cdots & \cdots & \cdots & \cdots & \mathbf{x}(N-1) \\
0 & \mathbf{x}(0) & \mathbf{x}(1) & \mathbf{x}(2) & \cdots & \cdots & \cdots & \mathbf{x}(N-2) \\
\vdots & \vdots & \vdots & \vdots & \vdots & \vdots & \vdots & \vdots \\
0 & \cdots & \cdots & \mathbf{x}(0) & \mathbf{x}(1) & \cdots & \cdots & \mathbf{x}(N-L-1)
\end{array}\right\}, \\
\left\{\begin{array}{cccccccc}
\mathbf{x}(1) & \mathbf{x}(2) & \cdots & \cdots & \cdots & \cdots & \mathbf{x}(N-1) & 0 \\
\mathbf{x}(0) & \mathbf{x}(1) & \mathbf{x}(2) & \cdots & \cdots & \cdots & \cdots & \mathbf{x}(N-1) \\
\vdots & \vdots & \vdots & \vdots & \vdots & \vdots & \vdots & \vdots \\
0 & \cdots & \mathbf{x}(0) & \mathbf{x}(1) & \cdots & \cdots & \cdots & \mathbf{x}(N-L)
\end{array}\right\} .
\end{gathered}
$$

If frame length $N$ is sufficiently large, the rows of $\mathcal{X}^{[q]}$ are linear independent with probability of 1 . Observing the block Toeplitz structure of the above matrices, the row rank of the intersection is $(K(L+1)-K)$. Therefore, the number of basis vectors of $\delta_{\mathfrak{Y}^{(1)}} \bigcap \delta_{\mathfrak{Y}^{(2)}}$ is also $(K(L+1)-K)$.

Following the similar verification procedure, it could be observed that the number of row basis vectors of

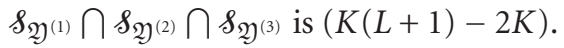

Moreover, the number of basis vectors of $\left\{\bigcap_{i=0}^{L} \delta_{\mathfrak{Y}^{(i)}}\right\}$ is $K$, which is equal to the number of row basis vectors for $\delta_{X}$. Hence, from (A.4), it is concluded that

$$
\diamond_{X}=\left\{\bigcap_{i=0}^{L} \diamond_{\mathfrak{Y}^{(i)}}\right\} .
$$

\section{ACKNOWLEDGMENT}

Part of the work described in this paper was presented during the Fourth IEEE International Workshop on Mobile and Wireless Communications Network, September 2002, Stockholm, Sweden.

\section{REFERENCES}

[1] A. J. Paulraj and C. B. Papadias, "Space-time processing for wireless communications," IEEE Signal Processing Magazine, vol. 14 , no. 6, pp. 49-83, 1997.

[2] C. Schlegel and Z. Bagley, Efficient processing for high-capacity MIMO channels, preprint, http://www.ee.ualberta.ca/ \%7eschlegel/publications.html.

[3] G. J. Foschini and M. J. Gans, "On limits of wireless communications in a fading environment when using multiple antennas," Wireless Personal Communications, vol. 6, no. 3, pp. 311-335, 1998. 
[4] G. J. Foschini, "Layered space-time architecture for wireless communication in a fading environment when using multielement antennas," Bell Labs Tech. Journal, vol. 1, no. 2, pp. 41-59, 1996.

[5] P. W. Wolniansky, G. J. Foschini, G. D. Golden, and R. A. Valenzuela, "V-BLAST: an architecture for realizing very high data rates over the rich-scattering wireless channel," in Proc. URSI International Symposium on Signals, Systems, and Electronics, pp. 295-300, Pisa, Italy, September-October 1998.

[6] T. H. Liew and L. Hanzo, "Space-time codes and concatenated channel codes for wireless communications," Proceedings of the IEEE, vol. 90, no. 2, pp. 187-219, 2002.

[7] V. Tarokh, N. Seshadri, and A. R. Calderbank, "Space-time codes for high data rate wireless communication: performance criterion and code construction," IEEE Transactions on Information Theory, vol. 44, no. 2, pp. 744-765, 1998.

[8] B. L. Hughes, "Differential space-time modulation," IEEE Transactions on Information Theory, vol. 46, no. 7, pp. 25672578, 2000

[9] B. L. Hughes, "Space-time group codes," in Proc. 34th Asilomar Conference on Signals, Systems, and Computers, vol. 1, pp. 699-704, Pacific Grove, Calif, USA, October-November 2000.

[10] J. Liu, J. Li, H. Li, and E. G. Larsson, "Differential space-code modulation for interference suppression,” IEEE Trans. Signal Processing, vol. 49, no. 8, pp. 1786-1795, 2001.

[11] J. Liu, E. G. Larsson, J. Li, and H. Li, "High-rate differential space-code modulation for interference suppression," in Proc. 3rd IEEE Signal Processing Workshops on Signal Processing Advances in Wireless Communications, pp. 283-286, Taoyuan, Taiwan, March 2001.

[12] B. M. Hochwald and W. Sweldens, "Differential unitary spacetime modulation,” IEEE Trans. Communications, vol. 48, no. 12, pp. 2041-2052, 2000.

[13] N. D. Sidiropoulos and R. S. Budampati, "Khatri-Rao spacetime codes,” IEEE Trans. Signal Processing, vol. 50, no. 10, pp. 2396-2407, 2002.

[14] Q. Ma, C. Tepedelenlioglu, and Z. Liu, "Differential spacetime-frequency coded OFDM with maximum diversity," in Proc. 37th Annual Conference on Information Sciences and Systems, Baltimore, Md, USA, March 2003.

[15] B. L. Hughes, "Further results on differential space-time modulation," in Proc. IEEE Sensor Array and Multichannel Signal Processing Workshop, pp. 163-167, Cambridge, Mass, USA, March 2000.

[16] A.-J. van der Veen, S. Talwar, and A. Paulraj, "A subspace approach to blind space-time signal processing for wireless communication systems," IEEE Trans. Signal Processing, vol. 45, no. 1, pp. 173-190, 1997.

[17] D. Gesbert, H. Bolcskei, D. A. Gore, and A. J. Paulraj, "MIMO wireless channels: capacity and performance prediction," in Proc. IEEE Global Communications Conference, vol. 2, pp. 1083-1088, San Francisco, Calif, USA, November 2000.

[18] Y. Wang, Y. C. Pati, Y. M. Cho, A. Paulraj, and T. Kailath, "A matrix factorization approach to signal copy of constant modulus signals arriving at an antenna array," in Proc. 28th Annual Conference on Information Sciences and Systems, pp. 178-183, Princeton, NJ, USA, March 1994.
Zhan Zhang received his B.S. degree in electronic engineering from Northwestern Polytechnic University, Xi'an, China, in 1990, and the M.A.S. degree from Beijing Institute of Technology, Beijing, China, in 1996. He is now working toward the Ph.D. degree at the Department of Electrical \& Computer Engineering, Dalhousie University, Halifax, Canada. His main research interests are in the areas of signal processing for digital communications, with emphases on multiuser detection, space-time channel coding and signal processing, and channel identification and equalization.

Jacek Ilow is an Associate Professor at the Department of Electrical and Computer Engineering, Dalhousie University, Halifax, Canada. He joined Dalhousie as an Assistant Professor in April 1997, and was promoted to the Associate Professor with tenure in July 2002. He received the B.Eng. degree in electronics from the Wroclaw University of Technology, Poland, in 1987 and the M.A.S. and Ph.D. degrees in electrical

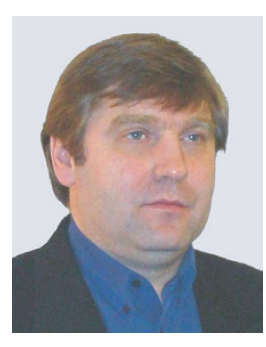
and computer engineering from the University of Toronto, Canada, in 1992 and 1996, respectively. From 1987 to 1988, he worked as a Researcher at the Wroclaw University of Technology in the Institute of Telecommunication and Acoustics. From December 1995 until March 1997, Dr. Ilow held an NSERC postdoctoral fellowship at the Defense Research Establishment, Ottawa, Ontario. His research interests lie in the areas of wireless networks, digital communications, and statistical signal processing. 